\title{
Neurodevelopmental Outcome after Hematopoietic Cell Transplantation in Inborn Errors of Metabolism: Current Considerations and Future Perspectives
}

\author{
Jaap Jan Boelens $s^{1,2,3}$ Peter M. van Hasselt ${ }^{3,4}$ \\ 1 Pediatric Blood and Marrow Transplantation Program, University \\ Medical Center Utrecht, Utrecht, The Netherlands \\ 2 Laboratory Translational Immunology, University Medical Center \\ Utrecht, Utrecht, The Netherlands \\ ${ }^{3}$ Sylvia Toth Center, University Medical Center Utrecht, Utrecht, The \\ Netherlands \\ ${ }^{4}$ Department of Metabolic Diseases, University Medical Center \\ Utrecht, Utrecht, The Netherlands
}

\begin{abstract}
Address for correspondence Jaap Jan Boelens, MD, PhD, Pediatric Blood and Marrow Transplantation Program, University Medical Center Utrecht, Utrecht, The Netherlands (e-mail: J.J.Boelens@umcutrecht.nl).
\end{abstract}

Neuropediatrics 2016;47:285-292.

\begin{abstract}
Keywords

- hematopoietic cell transplantation

- neurodevelopment

- indications

- gene therapy

Inborn errors of metabolism (IEM) comprise an assorted group of inherited diseases, some of which are due to disordered lysosomal or peroxisomal function and some of which might be improved following hematopoietic cell transplantation (HCT). In these disorders the onset in infancy or early childhood is typically accompanied by rapid deterioration, resulting in early death in the more severe phenotypes. Timely diagnosis and immediate referral to an IEM specialist are essential steps in optimal management. Treatment recommendations are based on the diagnosis, its phenotype, rate of progression, prior extent of disease, family values, and expectations, and the risks and benefits associated with available therapies, including HCT. International collaborative efforts are of utmost importance in determining outcomes of therapy for these rare diseases, and have improved those outcomes significantly over the last decades. In this review, we will focus on the neurodevelopmental outcomes after HCT in IEM, providing an international perspective on progress, limitations, and future directions.
\end{abstract}

\section{Introduction}

Inborn errors of metabolism (IEM) are a heterogeneous group of diseases caused by genetic defects in a wide array of metabolic pathways, including deficiencies in the production of lysosomal enzymes (lysosomal storage disease, LSDs) and abnormalities of peroxisomal function. Lysosomal enzymes are hydrolytic and catalyze the degradation of specific substrates within the acidic environment of lysosomes. Peroxisomes are subcellular organelles primarily involved in the metabolism of complex lipids (such as bile acids). The consequences of these

received

January 18, 2016

accepted after revision

April 19, 2016

published online

June 16, 2016

diseases are multisystemic, affecting bone integrity, growth and development, cardiopulmonary status, the airway, hearing and vision, neurological and cognitive function, and often result in premature death. Allogeneic-hematopoietic cell transplantation (HCT) has shown to be a treatment option for a selected group of patients with an IEM. Timely diagnosis and immediate referral to a "specialist in IEM," followed by a thorough evaluation by a multidisciplinary team, discussion with a multidisciplinary team including a transplant-physician, are essential steps. Treatment recommendations are based on: the disorder; its phenotype, including age at onset, rate of progression,

(c) 2016 Georg Thieme Verlag KG Stuttgart - New York
DOI http://dx.doi.org/ 10.1055/s-0036-1584602. ISSN 0174-304X. 
severity of clinical signs and symptoms; family values and expectations; and the risks and benefits associated with available therapies such as HCT.

To evaluate the efficacy of the various treatment modalities in these rare diseases, international collaborative efforts are critical. The collaborations started in late 1980s and have intensified over the past decade. Collaborative studies must distinguish early outcomes (transplant outcomes) from disease-specific outcomes in the longer term. Transplant outcomes are easier to study and identification of predictors that lead to engraftment failure or transplant mortality has resulted in really significant improvements. Assessment of long-term outcome is more difficult. The ability of HCT and the variables associated with $\mathrm{HCT}$, for example, graft-type and age at transplant to affect disease manifestations is more difficult. Although difficult, it is a key achievement of the HCT and IEM communities that such studies are now taking place. These studies are of utmost importance as the very purpose of HCT is to improve disease manifestations and improve quality and length of life.

This review will focus on neurodevelopmental outcome of HCT for lysosomal storage diseases and peroxisomal disorders as IEM. We will also discuss which diseases to transplant, when to transplant, and how to follow-up.

\section{Allogeneic HCT in IEM: What Is the Rationale and How Did We Get Here?}

In 1968 Fratantoni et al first established that substrate accumulation in the cells of patients with lysosomal enzyme deficiencies could be dramatically reduced by coculturing these cells with cells producing the missing enzyme. ${ }^{1}$ The ensuing principle of "cross-correction" was subsequently used to explore both exogenous enzyme replacement therapy and allogeneic HCT in numerous lysosomal diseases. As HCT also enables engraftment of donor-derived microglial cells in the brain-serving as local enzyme production units-this treatment, contrary to enzyme replacement therapies (ERT), has the potential to treat central nervous system (CNS) manifestations. This strategy has been tested in various disorders, including mucopolysaccharidosis type-1, Hurler phenotype (MPS-1H) and several leukodystrophies, in particular metachromatic leukodystrophy (MLD), X-linked adrenoleukodystrophy (X-ALD), and globoid cell leukodystrophy (GLD; or Krabbe disease). HCT has since become the standard of care in a selected group of disorders (e.g., MPS-1H, early XALD) and since the first HCT in an MPS- $1 \mathrm{H}$ patient, ${ }^{2}$ over 2,000 patients with an IEM have been transplanted ( $\mathbf{F i g . 1}$. ${ }^{9}{ }^{9}$ Treatment recommendations may vary significantly, even within a particular diagnosis. These recommendations reflect the type of disorder, its predicted phenotype, the extent of disease progression, family values, and expectations and aim to balance the cumulative risks and benefits associated with available therapies.

Initially, HCT was associated with significant mortality due to high rates of graft-failure and transplantation-related mortality. International collaborations have identified predictor for these mortalities, resulting in engrafted survival
Allogeneic Transplants for Inborn Errors of Metabolism Registered with CIBMTR, 1980-2013

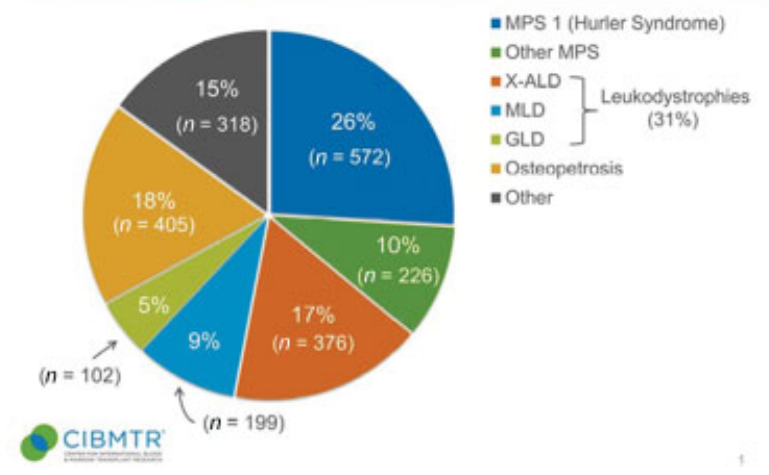

Fig. 1 Since early 1980s over 2,000 HCT in IEM have been performed. Here, the distribution of the various diseases/disease-groups is shown (CIBMTR database 2013). HCT, hematopoietic cell transplantation; IEM, inborn errors of metabolism. (Adapted from Boelens J], Orchard PJ, Wynn RF. Transplantation in inborn errors of metabolism: current considerations and future perspectives. Br J Haematol 2014;167(3):293-303). ${ }^{9}$

rates of over $90 \%$ nowadays. ${ }^{3-5}$ HCT for IEM is performed using donor-derived hematopoietic stem cells obtained from bone marrow (BM), growth factor mobilized peripheral blood $(\mathrm{PB})$, and the last decade predominantly from umbilical cord blood (CB). Allogeneic HCT has become much safer due to the availability of better-matched cords, enhanced techniques for HLA matching and increased inventory, individualized conditioning regimens and supportive care. Currently, for patients with nonmalignant disease such as IEM, the rate of HCT-related complications such as infections and "graftversus-host disease" (GvHD), are relatively low, especially chronic-GvHD $(<10 \%)^{3,6}$

\section{Which Patients to Transplant?}

Although HCT has proven to influence the natural course of a variety of IEM significantly not all diseases and disease phenotypes benefit from HCT. The currently accepted IEM indications (including optional or investigational diagnoses) for HCT are described in - Table 1.

Transplant decisions require balancing potential risks and benefits. Patients with a milder phenotype and/or in those patients that are earlier in the course of their disease are more likely to benefit. Early recognition is therefore of utmost importance as much of the morbidity associated with the disease itself is not reversed by HCT. Treatment of the CNS with HCT is primarily mediated by the engraftment of donor-derived microglial cells in the brain. As it takes several months to replace sufficient microglia with those originating from the donor, there may be a delay in benefits of transplant to the CNS. As a consequence, diseases affecting the brain that are rapidly progressive (e.g., infantile GLD) are much more difficult to treat with HCT. HCT may only offer clinical benefit in these diseases if established presymptomatically, for example, in families with affected siblings. As short-term results of HCT improve (higher engrafted survival rates) and its overall risk is thereby reduced, it is now 
Table 1 Inborn errors of metabolism for which HCT may be indicated

\begin{tabular}{|c|c|c|c|}
\hline Disorder & Enzyme/protein & Indication & Comments \\
\hline \multicolumn{4}{|l|}{ Mucopolysaccharidosis } \\
\hline Hurler (MPS-1H) & $\alpha$-L-iduronidase & Standard & \\
\hline Hurler/Scheie (MPS-1H/S) & $\alpha$-L-iduronidase & Option & ERT first-line therapy \\
\hline Scheie (MPS-1S) & $\alpha$-L-iduronidase & Option & ERT first-line therapy \\
\hline Hunter: Severe (MPS-2A) & Iduronate-2-sulfatase & Investigational & Only early or asymptomatic \\
\hline Hunter: Attenuated (MPS-2B) & Iduronate-2-sulfatase & Option & ERT first-line therapy \\
\hline Maroteaux-Lamy (MPS-6) & Arylsulfatase B & Option & ERT first-line therapy \\
\hline Sly (MPS-7) & $\beta$-glucuronidase & Option & \\
\hline \multicolumn{4}{|l|}{ Leukodystrophies } \\
\hline X-ALD, cerebral & ALD protein & Standard & Not for advanced disease \\
\hline MLD: Early infantile & Arylsulfatase A & No & \\
\hline MLD: Late-infantile/juvenile & Arylsulfatase A & Option/standard & Gene-therapy trial open (Milan) \\
\hline MLD: Adult onset & Arylsulfatase A & Standard & Only early or asymptomatic \\
\hline GLD: Early onset & Galactocerebrosidase & Standard & $\begin{array}{l}\text { Neonate, screening diagnosis, } \\
\text { or second case in known family; } \\
\text { not for advanced disease }\end{array}$ \\
\hline GLD: Late onset & Galactocerebrosidase & Option & \\
\hline \multicolumn{4}{|c|}{ Glycoprotein metabolic and miscellaneous disorders } \\
\hline Fucosidosis & Fucosidase & Option & \\
\hline$\alpha$-mannosidosis & a-Mannosidase & Option & \\
\hline Aspartylglucosaminuria & Aspartylglucosaminidase & Option & \\
\hline Farber & Ceraminidase & Option & \\
\hline Tay-Sachs: Early onset & Hexosaminidase $\mathrm{A}$ & No & \\
\hline Tay-Sachs: Juvenile & Hexosaminidase $\mathrm{A}$ & Option & In known family \\
\hline Sandhoff: Early onset & Hexosaminidase $A$ and $B$ & No & $\begin{array}{l}\text { Neonate, screening diagnosis, } \\
\text { or second case }\end{array}$ \\
\hline Sandhoff: Juvenile & Hexosaminidase $A$ and $B$ & Option & \\
\hline Gaucher I (nonneuronopathic) & Glucocerebrosidase & Unknown & ERT first-line therapy \\
\hline Gaucher II (acute neuronopathic) & Glucocerebrosidase & Option & \\
\hline Gaucher III (subacute neuronopathic) & Glucocerebrosidase & Unknown & Limited benefit of ERT \\
\hline Pompe & Glucosidase & Unknown & ERT available \\
\hline Niemann-Pick: Type A & Acid sphingomyelinase & Investigational & \\
\hline Niemann-Pick: Type B & Acid sphingomyelinase & Unknown & ERT first-line therapy \\
\hline Niemann-Pick: Type C & Cholesterol trafficking & Option & Only early or asymptomatic \\
\hline Wolman syndrome & Acid lipase & Option & May be viewed as standard \\
\hline Multiple sulfatase deficiency & Sulfatases & Investigational & Not in advanced disease \\
\hline $\begin{array}{l}\text { Mitochondrial neurogastrointestinal } \\
\text { encephalomyopathy (MNGIE) }\end{array}$ & Thymidine phosphorylase & Option & \\
\hline
\end{tabular}

Abbreviations: ERT, enzyme replacement therapies; HCT, hematopoietic cell transplantation.

Notes: Table does not include diseases where HCT is not indicated/contraindicated.

Standard: HCT applied routinely. Considerable published research evidence from registries and institutions demonstrates efficacy. Delayed diagnosis and/or advanced disease may preclude transplant for individual patients.

Option: HCT is effective but other therapy is increasingly considered first choice. Insufficient published evidence for HCT to be considered standard. Investigational: Possible a priori reason for HCT. Further published evidence needed to support the use of HCT in clinical practice. 
appropriate to consider whether diseases of milder phenotype might be considered for HCT, as the alternative is weekly 4 to 6 -hour infusions of enzyme, and data suggest better metabolic correction after unrelated HCT compared with intravenous enzymes. ${ }^{7}$ This might include milder LSD currently managed with ERT, such as Hurler-Scheie, MPS-6 but also (attenuated) MPS-2.

Much of the literature describing outcomes with HCT in any particular disease is outdated: for example, it is said that HCT is ineffective in MPS-2 (Hunter), but such a statement is derived from HCT experience in symptomatic children using sibling donor grafts (which may often be carriers). The outcomes may be different in those same patients had it been performed earlier and using an unrelated cord blood donor, where enzyme delivery would have been optimized as shown in some recent studies. ${ }^{3,4}$ Thus, some of these HCT questions must be re-evaluated in this modern era. Also, here collaboration between centers for these re-evaluations is of importance since these diseases are rare and sometimes even ultrarare.

The role of HCT in a selected group of LSD and cerebral-ALD (C-ALD) is well established and is discussed in more detail below especially focusing on the neurodevelopmental outcomes. The role of HCT in other metabolic disease is less clear.

\section{What Are the Neurodevopmental Outcomes of HCT in IEM?}

Here, we describe the outcomes of the most frequently transplanted diseases-groups: Leukodystrophies (31\%) and MPS-1H (27\%, - Fig. 1).

\section{Leukodystrophies}

GLD or Krabbe disease (incidence, 1:100,000), is a recessive disorder caused by defects in the galactocerebrosidase. Over $80 \%$ of cases of GLD present in infancy, with irritability, poor feeding, and progressive loss of motor function; the majority of these patients expire before 2 years of age. It is generally accepted that there is no role for HCT in symptomatic patients with the classic infantile phenotype. In patients identified and treated very early in life, either due to a positive family history or through newborn screening, HCT alters the course of the disease. ${ }^{8}$ In these patients, cognitive function is relatively spared, but significant loss of gross motor function is still being observed post-HCT (although less clear in attenuated phenotypes). The relative contribution of peripheral demyelination to motor-related dysfunction is difficult to assess, but it is clear that nerve conduction abnormalities are present in GLD post-HCT. The later onset forms of GLD progress less quickly, providing an opportunity longer time window for HCT. Later onset forms of GLD may not only be stabilized, but can potentially improve following HCT (reviewed recently ${ }^{9}$ ).

MLD, also recessive in inheritance (1 in 40,000) is caused by a defect in the arylsulfatase $\mathrm{A}$ (ARSA) gene. In this disorder, sulfatides accumulate in myelin-producing cells, resulting in both central and peripheral demyelination. ARSA testing is important in establishing the diagnosis, however, pseudode- ficiency states exist in which measured ARSA levels are low. Urinary sulfatide excretion is only elevated if ARSA is really deficient, urine sulfatide testing is therefore, necessary to confirm the diagnosis of MLD. MLD is categorized based on age at onset of clinical manifestations: late-infantile (younger than 2 years), juvenile (3-16 years), and adult forms. Lateinfantile MLD is the most common form and is characterized by progressive motor dysfunction resulting in a gradual loss of the ability to walk, stand and sit, dysarthria, swallowing difficulties, an inability to handle secretions, and early death ( $<4$ years of age). There is no role for HCT in symptomatic late-infantile MLD. ${ }^{8}$ Patients with late-infantile disease that proceeded to HCT presymptomatically achieved some stabilization of disease, but eventually severe motor difficulties were observed in most, if not all, primarily due to peripheral nerve dysfunction. ${ }^{10}$ The asymptomatic late-infantile group is also the target of recent gene-therapy initiatives and has the capacity to deliver more enzyme than standard allogenicHCT. First clinical evidence of efficacy has been shown recently. ${ }^{11}$ In attenuated forms of the disease a greater opportunity exists to impact the disease process with HCT. It has been reported that patients with juvenile disease transplanted while still asymptomatic attain much better outcomes. ${ }^{10}$ Patients with adult form of MLD (20\% of cases), which can become apparent as late as the sixth or seventh decade, have less motor findings, but may be emotionally labile and have difficulties in executive function, progressive dementia, psychosis, and drug abuse. Although limited data available there appears to be a role for allogeneic HCT for adult-onset disease (reviewed recently ${ }^{9}$ ).

ALD: In 1976 it was demonstrated that very long chain fatty acids (VLCFAs) accumulate in the brain and adrenal tissue of patients with ALD. ${ }^{12,13}$ The inability to degrade VLCFA is due to mutations in the $A B C D 1$ gene, encoding a peroxisomal membrane protein. ${ }^{14,15}$ ALD is an X-linked in inheritance $(1$ in 17,000) and clinical manifestations of the disease are highly variable. The most severe phenotype of ALD is the cerebral form (C-ALD), which is found in 35 to $40 \%$ of individuals with ALD by age 20. Cerebral ALD is an acute, inflammatory, demyelinating condition. In almost all (95\%) cases, C-ALD is associated with progressive neurological deterioration and ultimately death within several years. CALD is rare before 4 years of age, the median age in which cerebral manifestations occur is at 7 years. Since the first report by Aubourg et al on a beneficial effect of HCT in a boy with early-stage C-ALD in $1990^{16}$ additional experience has established allogeneic HCT as the standard of care for early CALD. Until now the exact mechanism of action remains unclear, but it is thought that chemotherapy and immune suppression associated with HCT controls neuroinflammation. Presumably, donor microglial cells provide support to the ALD oligodendrocyte. The Loes magnetic resonance imaging (MRI) scoring system, which provides a numeric score based on the number of areas of the brain with evidence of demyelination, ${ }^{17,18}$ is a predictor of survival, with higher mortality rates in advanced patients: those with higher scores (>9) have worse outcomes. ${ }^{6}$ Unfortunately, most patients are not diagnosed as having C-ALD until they develop clinically 
evident neurological changes, thus are having already advanced disease when HCT is considered. Presently, these patients are no longer eligible for treatment with HCT as mortality rates associated with transplant are high and disease burden after transplant is very high. Novel strategies such as using a reduced intensity regimen and antioxidative therapy, may change this recommendation in the future. ${ }^{6}$ An intriguing question regarding the role of HCT in ALD is whether HCT alters the natural history adrenomyeloneuropathy (AMN), a late-onset ALD disease complication. AMN affects the spinal cord, is noninflammatory and results in a slow progression of motor-related limitations beginning in the third decade of life. ${ }^{15}$ Recently, early reported success of gene therapy (autologous gene-transduced HCT) has been published and a second trial recently has started. ${ }^{19}$ Important will be the comparison between patients receiving allogenicHCT and gene-transduced autologous HCT, not only for the short (transplant)-term outcomes, but more importantly on the long-term outcomes.

\section{Hurler Syndrome, MPS-1H}

Hurler syndrome (MPS-1H), the most severe phenotype of $\alpha$ L-iduronidase deficiency, is an autosomal recessive disorder characterized by progressive accumulation of glycosaminoglycans (GAGs). Hurler and other phenotypes of MPS-1Scheie (attenuated) and Hurler-Scheie (MPS-1H/S, intermediate) represent a continuous clinical spectrum. Accumulation of GAGs results in progressive, multi-system dysfunction associated with premature death. Over 600 HCTs have been performed worldwide for children with MPS-1H since 1980 (European Society for Blood and Marrow Transplantation/ Center for International Blood and Marrow Transplantation Research (EBMT/CIBMTR) registry), making it the most commonly transplanted IEM (-Fig. 1).

HCT for children with MPS-1H has been shown to increase life expectancy and improve the clinical manifestations of disease. ${ }^{4,20}$ HCT must be performed early in the disease course as any disease-related complications, particularly neurocognitive dysfunction, are irreversible. Donor-cell engraftment after HCT has resulted in the rapid reduction of obstructive airway symptoms and hepatosplenomegaly. ${ }^{20}$ Hearing, vision, and linear growth, improve in most cases. Hydrocephalus is either prevented or stabilized and the otherwise expected cardiovascular pathology is altered beneficially after HCT. Although cerebral damage already present before HCT appears to be irreversible, successful HCT is able to prevent progressive psychomotor deterioration and permit continued neurodevelopment., 4

International collaborative studies identified predictors for graft-failure associated with poor "event-free survival" rates; T-cell depleted grafts and reduced intensity conditioning, while busulfan with therapeutic drug monitoring (targeting to a myeloablative exposure) appeared to be a predictor for higher "event-free survival" in comparison to prior experiences. $^{3,22}$ These data have led to an EBMT transplant protocol/guideline (EBMT/European Hematology Association (EHA) handbook 2008 and 2012). ${ }^{23}$ These guidelines included a standardized busulfan/cyclophosphamide conditioning regimen and the use of $\mathrm{CB}$ as a preferred graft-source, second only to noncarrier-matched sibling BM. This transplant protocol with well-matched grafts resulted in a significantly improved engrafted survival rate of over 90\% in larger experienced HCT-centers specialized in transplanting MPS patients, who also have standardized long-term follow-up programs. ${ }^{5}$ Recently, the conditioning was modified to busulfan with pharmacokinetic monitoring: targeting to myeloablative busulfan exposure) + fludarabine (EBMT/EHA handbook 2012). Recent conditioning comparisons (BuCy vs. FluBu) suggest similar HCT outcomes, but with reduced toxicity. $^{5,24}$

Over the past decade, unrelated $\mathrm{CB}$ has been used with increasing frequency as a graft source for HCT in children with an IEM. CB offers several advantages over BM or PB including reduced time to HCT, greater tolerance for HLA-mismatch, lower incidence, and severity of GvHD, and reduced likelihood of transmitting viral infections. Recent collaborative studies suggest that highest event-free survival (EFS) rates are achieved in patients receiving an identical matched sibling donor or an identical (6/6) unrelated cord blood, followed by $5 / 6$ matched $\mathrm{CB}$ (or a $4 / 6$ matched $\mathrm{CB}$ donor with high-cell dose) or 10/10 matched unrelated donor. Interestingly, almost all $\mathrm{CB}$ recipients had full-donor chimerism associated with normal enzyme levels, while mixed-chimerism was more frequently seen in matched sibling and matched unrelated donor (MUD) donors. ${ }^{3}$ It is also important to recognize that most matched sibling donors are carriers, influencing posttransplant enzyme levels. Lower enzyme levels appear to be important for long-term outcomes, including neurocognitive outcomes: patients with normal enzymes (in leukocytes according to local reference range) have much better outcomes: better growth, less orthopedic interventions less/no respiratory support. ${ }^{4,20}$

Survival of MPS-1H patients has significantly improved last decade; however, the major limitation is substantial morbidity due to "residual disease burden"; primarily musculoskeletal features that often require orthopedic surgical interventions. $^{4,20,21}$ Use of improved and reduced toxicity HCT techniques at an earlier age and the achievement of full donor-chimerism with normalization of enzyme activity may enhance outcomes. Newborn screening may prove a major step forward in early identification of individuals with MPS$1 \mathrm{H}$.

\section{When to Transplant?}

It is clear that in IEM the outcomes (both transplant outcomes and late outcomes) are superior in patients in whom transplant is performed early. This has thus far been demonstrated for several diseases, including MPS-1H (Hurler disease), MLD, C-ALD, and Krabbe disease..$^{3,6,8,10}$ It will likely hold true for other (ultrarare) IEM for which HCT has a role.

Newborn screening is emerging as an option for these disorders; previously the median age at transplant for Hurler was $\sim 16$ months of age, ${ }^{3}$ as most children are diagnosed based on clinical signs and symptoms. The age at transplant will likely be significantly reduced as neonatal screening is 
implemented (which is agreed upon in several countries; including the Netherlands). The test(s) need to be specific, cost-effective, be able to identify disease-causing mutations and be able to offer a reliable prediction of the phenotype to minimizing anxiety and uncertainty in families. Of course the introduction of such screening should be prospectively evaluated in a standardized manner to evaluate the benefits of intervention.

In C-ALD, as discussed above, intervention with HCT is only offered when demyelination is documented based on MRI changes. Neonatal screening for ALD has already begun in New York, and will likely expand in the near future (including the Netherlands). This will allow boys to be identified early, providing them the best chance for performing HCT quickly as cerebral disease is identified and allowing detection of adrenal failure so that life-saving hormone replacement therapy can be given. Currently, HCT is not performed in boys without documented MRI changes because of the risk of HCT, and due to our inability to identify those who will ultimately develop cerebral disease. This paradigm is likely to change when evidence proves that HCT alters the course of AMN, as discussed above.

\section{How to Transplant a Patient with an IEM?}

Engraftment has been shown to require myeloablative conditioning (which means maximal intensity to make space in the bone marrow) most commonly utilizing full-dose targeted busulfan. ${ }^{3,22,25}$ In dedicated, experience centers, transplanting patients with IEM, the engrafted survival rates are above 90 to $95 \%$ using busulfan-based myeloablative conditioning. ${ }^{5}$ If in upcoming years protocols can be developed with even further reduction of the toxicity associated with equivalent engraftment as the current myeloablative conditioning protocols this will be advantageous, especially when this will give benefits in regards to fertility and normal endocrine function after HCT. Agents, such as, treosulfan are considered to be less toxic (but is proven to be less ablative and systematic pharmacokinetic (PK) and pharacodynamic (PD) studies are lacking), there are data that clearly suggest that busulfan may provide superior microglial engraftment (essential for earlier and higher delivery of the missing enzyme in the brain). The replacement of cyclophosphamide for fludarabine combined with exposure-targeted busulfan has proven to be as effective (including absence of mixed chimerism, which is also more frequently seen in treosulfan-based regimens) but significantly less toxic. ${ }^{24}$ Based on current literature the recommended conditioning regimen and cell source hierarchy is described in -Table 2 .

The enzyme dose delivered by HCT is important in patient's "late outcomes" after HCT. Children that are fully engrafted from unrelated donors or noncarrier-related donors have typically high enzyme levels and need less orthopedic surgery and grow better. ${ }^{4}$ Several lines of evidence suggest that autologous cells that are gene-modified to express the deficient enzyme may further improve outcomes compared with the wild type donor. ${ }^{3,11}$ Furthermore, gene therapy has the capacity to enhance enzyme secretion not only by increasing the gene copy number but also by re-regulating gene expression so that it occurs in monocytic and other mature blood cells. As the enzyme level has shown to be a predictor of long-term outcomes (e. g., skeletal disease $)^{4}$ higher enzymes achieved by genetherapy may influence the long-term outcomes positively in the future. Comparing the gene-therapy outcomes with current practice (HCT) is of course important to prove possible superiority.

\section{What Are Alternative Treatment Options for Patients with an IEM?}

In addition to supportive care of disease manifestations and HCT alternative therapies are increasingly available for these conditions. The success of Cerezyme (imiglucerase, genzyme) for the treatment of Gaucher disease has led to the development of similar therapies for other LSDs. Fabry disease, MPS I, II, VI, and Pompe disease all have ERT and many more enzymes are in various stages of clinical development. This approach however has several limitations; not all patients are suitable for treatment, some organs or tissues (e.g., lung, liver, spleen) are more amenable to correction than others (e.g., skeletal, CNS). In addition, there are problems gauging efficacy in this group of highly variable disorders. Furthermore, there is a burden of weekly (or even more frequent) infusions, development of (neutralizing) antibodies to the recombinant proteins and ERT will not penetrate of the CNS which makes ERT not an effective treatment option for patients with CNS disease.

Other alternative therapies are being developed such as oral small molecules acting as either inhibitors of substrate accumulation or as chaperones to misfolded proteins. With this approach, or combined strategies it is hoped that there is some or better effect on CNS disease, but all are too early to change decision making.

As already discussed above, gene therapy approaches have translated from the laboratory to the clinic; the first trials are accruing patients in C-ALD and MLD. ${ }^{11,19}$ In the very near future also trials in other LSDs will start: for example, MPS-3, MPS-1.

\section{How to Follow-Up and Provide Care to Patients Enouncing Residual Disease Burden}

Structured multidisciplinary follow after HCT is an essential element of any transplant program as it provides robust feedback on the decisions made during the process. Given the rarity of these disorders and the heterogeneity within each disorder (age at transplant, disease severity, etc.) the risks and benefits may be unclear at the time of transplant. It is increasingly recognized that residual disease burden may be substantial despite HCT. ${ }^{4}$ The residual disease burden is multifaceted, often involving several organ systems, and structured and multidisciplinary follow-up ensures that the various aspects are recognized and treated at an early stage. Also, it allows recognition of previously unsuspected longterm morbidity, which may be amenable to treatment. ${ }^{26}$ In 
Table 2 Donor hierarchy and conditioning

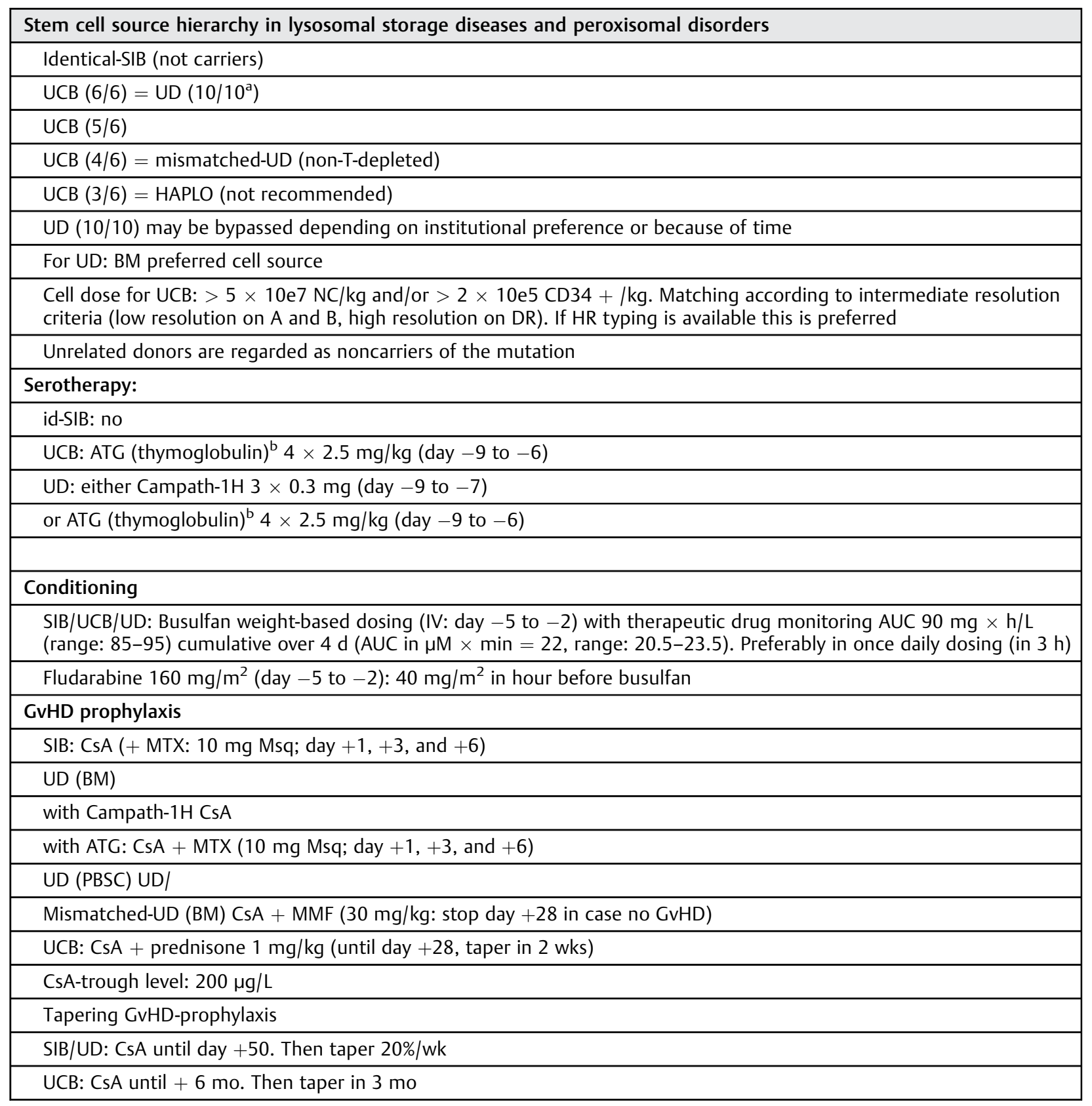

Abbreviations: ATG, anti-thymocyte globuline; AUC, area under the curve; Campath-1H, Alemtuzumab; CsA, ciclosporine-A; GvDH, graft-versus-host disease; HLA, human leukocyte antigen; MMF, mofitil-mycofenolaat; mo, month; Msq, meter square; MTX, methotrexate; NC, nucleated cells; HR, high resolution; PBSC, peripheral blood stem cells; UCB, unrelated cord blood; UD, unrelated donor; SIB, identical sibling donor; BM, bone marrow. ${ }^{\mathrm{a}} 10 / 10 \mathrm{HLA}$ matched on high resolution typed.

${ }^{\mathrm{b}}$ ATC dose for $>25 \mathrm{~kg}: 3 \times 2.5 \mathrm{mg} / \mathrm{kg}$ and $>50 \mathrm{~kg}: 2 \times 2.5 \mathrm{mg} / \mathrm{kg}$ (start day -9$)$.

case of research/developmental indication for HCT, structured multidisciplinary follow-up will help determining whether the indication is proving valid.

\section{Summary}

HCT is an effective treatment option for a selected group of inborn errors of metabolism: for example, some LSDs, and peroxisomal disorders. The HCT outcomes as well as the longterm perspective have positively changed over the last de- cade. Intense international collaboration has resulted in these better outcomes due to the identification of predictors of outcomes. It however remains important to continue with these tight collaborations as novel therapies are being developed to further improve the outcomes. In summary:

- Full-intensity myeloablative conditioning has been required for sustained donor cell engraftment in patients with IEM. This is best achieved by exposure-targeted busulfan in combination with immune suppressive drugs 
(fludarabine or cyclophosphamide) and serotherapy (e.g., antithymocyte globulin/Campath [ATG and Campath: Genzyme, Cambridge, Massachusetts, United States])

- Engrafted survival rates have significantly improved and in experienced centers might be expected to be over $90 \%$

- Cord blood as a donor cell source gives better chimerism than bone marrow or peripheral blood after myeloablative conditioning

- Structured multidisciplinary follow-up after HSCT is an essential element in the care of these patients not only to optimize treatment of residual disease, but also to delineate efficacy and to identify clinical targets for improvement

- The primary goal of HCT is optimizing the functional outcomes of these patients, and their "quality of life." Variables that can be addressed to increase efficacy of therapy, including:

\section{- Age at transplant: The earlier the better}

- Levels of enzyme delivered to recipient tissue by engrafted donor leukocytes, which are currently best achieved after myeloablative cord blood transplantation. In the future, the use of gene-therapy protocols may result in supranormal enzyme levels, which may have an impact on the long-term outcomes of these patients $\circ$ Phenotype of disease: The milder the more promising

\section{References}

1 Fratantoni JC, Hall CW, Neufeld EF. Hurler and Hunter syndromes: mutual correction of the defect in cultured fibroblasts. Science 1968;162(3853):570-572

2 Hobbs JR, Hugh-Jones K, Barrett AJ, et al. Reversal of clinical features of Hurler's disease and biochemical improvement after treatment by bone-marrow transplantation. Lancet 1981;2(8249):709-712

3 Boelens JJ, Aldenhoven M, Purtill D, et al; Eurocord; Inborn Errors Working Party of European Blood and Marrow Transplant group; Duke University Blood and Marrow Transplantation Program; Centre for International Blood and Marrow Research. Outcomes of transplantation using various hematopoietic cell sources in children with Hurler syndrome after myeloablative conditioning. Blood 2013;121(19):3981-3987

4 Aldenhoven M, Wynn RF, Orchard PJ, et al. Long-term outcome of Hurler syndrome patients after hematopoietic cell transplantation: an international multicenter study. Blood 2015; 125(13):2164-2172

5 Aldenhoven M, Jones SA, Bonney D, et al. Hematopoietic cell transplantation for mucopolysaccharidosis patients is safe and effective: results after implementation of international guidelines. Biol Blood Marrow Transplant 2015;21(6): 1106-1109

6 Miller WP, Rothman SM, Nascene D, et al. Outcomes after allogeneic hematopoietic cell transplantation for childhood cerebral adrenoleukodystrophy: the largest single-institution cohort report. Blood 2011;118(7):1971-1978

7 Wynn RF, Wraith JE, Mercer J, et al. Improved metabolic correction in patients with lysosomal storage disease treated with hematopoietic stem cell transplant compared with enzyme replacement therapy. J Pediatr 2009;154(4):609-611

8 Escolar ML, Poe MD, Provenzale JM, et al. Transplantation of umbilical-cord blood in babies with infantile Krabbe's disease. N Engl J Med 2005;352(20):2069-2081
9 Boelens JJ, Orchard PJ, Wynn RF. Transplantation in inborn errors of metabolism: current considerations and future perspectives. Br J Haematol 2014;167(3):293-303

10 Martin HR, Poe MD, Provenzale JM, Kurtzberg J, Mendizabal A, Escolar ML. Neurodevelopmental outcomes of umbilical cord blood transplantation in metachromatic leukodystrophy. Biol Blood Marrow Transplant 2013;19(4):616-624

11 Biffi A, Montini E, Lorioli L, et al. Lentiviral hematopoietic stem cell gene therapy benefits metachromatic leukodystrophy. Science 2013;341(6148):1233158

12 Igarashi M, Schaumburg HH, Powers J, Kishmoto Y, Kolodny E, Suzuki K. Fatty acid abnormality in adrenoleukodystrophy. J Neurochem 1976;26(4):851-860

13 Schaumburg HH, Powers JM, Raine CS, et al. Adrenoleukodystrophy: a clinical, pathological and biochemical study. Adv Exp Med Biol 1976;68:379-387

14 Moser HW. Clinical and therapeutic aspects of adrenoleukodystrophy and adrenomyeloneuropathy. J Neuropathol Exp Neurol 1995;54(5):740-745

15 Moser HW, Bergin A, Naidu S, Ladenson PW. Adrenoleukodystrophy. Endocrinol Metab Clin North Am 1991;20(2): 297-318

16 Aubourg P, Blanche S, Jambaqué I, et al. Reversal of early neurologic and neuroradiologic manifestations of X-linked adrenoleukodystrophy by bone marrow transplantation. N Engl J Med 1990;322(26):1860-1866

17 Loes DJ, Stillman AE, Hite S, et al. Childhood cerebral form of adrenoleukodystrophy: short-term effect of bone marrow transplantation on brain MR observations. AJNR Am J Neuroradiol 1994;15(9):1767-1771

18 Loes DJ, Fatemi A, Melhem ER, et al. Analysis of MRI patterns aids prediction of progression in X-linked adrenoleukodystrophy. Neurology 2003;61(3):369-374

19 Cartier N, Hacein-Bey-Abina S, Bartholomae CC, et al. Hematopoietic stem cell gene therapy with a lentiviral vector in X-linked adrenoleukodystrophy. Science 2009;326(5954): 818-823

20 Aldenhoven M, Boelens JJ, de Koning TJ. The clinical outcome of Hurler syndrome after stem cell transplantation. Biol Blood Marrow Transplant 2008;14(5):485-498

21 Staba SL, Escolar ML, Poe M, et al. Cord-blood transplants from unrelated donors in patients with Hurler's syndrome. N Engl J Med 2004;350(19):1960-1969

22 Boelens JJ, Wynn RF, O'Meara A, et al. Outcomes of hematopoietic stem cell transplantation for Hurler's syndrome in Europe: a risk factor analysis for graft failure. Bone Marrow Transplant 2007;40(3):225-233

23 Boelens JJ, Bierings M, Wynn RF. HSCT for inborn errors of metabolism. In: Apperley J, Carreras E, Gluckman E, Masszi T, eds. The 2012 Revised Edition of the EBMT-ESH Handbook on Haematopoietic Stem Cell Transplantation. Chap 20. EBMTESH. 2012:558-571

24 Bartelink IH, van Reij EML, Gerhardt CE, et al. Fludarabine and exposure-targeted busulfan compares favorably with busulfan/ cyclophosphamide-based regimens in pediatric hematopoietic cell transplantation: maintaining efficacy with less toxicity. Biol Blood Marrow Transplant 2014;20(3):345-353

25 Bartelink IH, Boelens JJ, Bredius RGM, et al. Body weightdependent pharmacokinetics of busulfan in paediatric haematopoietic stem cell transplantation patients: towards individualized dosing. Clin Pharmacokinet 2012;51(5): 331-345

26 Langereis EJ, Borgo A, Crushell E, et al. Treatment of hip dysplasia in patients with mucopolysaccharidosis type I after hematopoietic stem cell transplantation: results of an international consensus procedure. Orphanet J Rare Dis 2013; 8(1):155 